\title{
Internet of Things and Healthcare Analytics for Better Healthcare Solution: Applications and Challenges
}

\author{
Zuraida Abal Abas ${ }^{1}$, Zaheera Zainal Abidin ${ }^{2}$, Ahmad Fadzli Nizam Abdul Rahman ${ }^{3}$, Hidayah Rahmalan ${ }^{4}$, Gede \\ Pramudya $^{5}$, Mohd Hakim Abdul Hamid ${ }^{6}$ \\ Fakulti Teknologi Maklumat \& Komunikasi, \\ Universiti Teknikal Malaysia Melaka, \\ 76100 Durian Tunggal, Melaka, Malysia
}

\begin{abstract}
The total number of population in the world will keep on increasing. This will eventually pose challenges towards quality of life for example issues related to healthcare. Hence, a proper solution needs to be devised in order to face the challenges. Internet of Things (IoT), which is one of the digital technologies, that is becoming a trend now can offer promising solution. This paper serves as a short communication in introducing IoT and its application in healthcare domain as well as the analytics combined with the technology. Some examples are presented according to the categories of the application. It must be noted that the analytics play an important role in making the IoT healthcare as a comprehensive solution. At the end of the paper, challenges in making this digital as an accessible solution is discussed.
\end{abstract}

Keywords-Internet of things; analytics; healthcare; applications; challenges

\section{INTRODUCTION}

Today, as recorded in world population clock, the total number of world population is about 7.6 billion in 2018 [1]. The total world population will keep on increasing as illustrated in Figure 1 [1]. The population growth is driven by several factors such as fertility rate, life expectancy (mortality) and migration [1]-[3]. Although the growth rate is decreasing or slower compared to previous time, the total number of world population will be projected to reach at about 9.8 billion in 2050, as projected by the United Nation [4]. This is actually a huge amount of number. Imagine the world is full with huge number of people; there must be several implications. Among the implications will definitely have a strong impact on quality of life.

Having the term 'quality of life', it is a normal setting to relate it with theory of Maslow's Hierarchy of Needs in mind as illustrated in Figure 2. The diagram of Maslow's Hierarchy of Needs is not an alien to most people, which has been seeing it from young age. In general, there are five levels of needs: physiological, safety, belonging, esteem and finally selfrealization. The interest of this paper is to highlight the health component that belongs to the safety level. The health component in the safety level usually brings together unity among various stakeholders, practitioner, and industries across the globe in order to bring innovative health solution.

It is worth to highlight that the quality of life is one of the issues that need to be handled in efficient way while facing growing population phenomenon. Again, one of the domains that are very related to quality of life is healthcare service. It must be noted that the growing population will somehow create new and lasting challenges for healthcare worldwide [5] In order to face these challenges, the emerging digital technology such as machine learning, cloud computing, Artificial Intelligence and Internet of Things (IoT) should be utilized. The world will benefit a lot more when utilizing digital technology especially in a healthcare setting.

This paper serves as a short communication in introducing IoT and its application in healthcare domain as well as the analytics combined with the technology. Section II presents the revolution of digital technology. Section III presents IoT for healthcare application while section IV presents the analytics and its role in IoT applications. Finally section V presents discussion and conclusion.

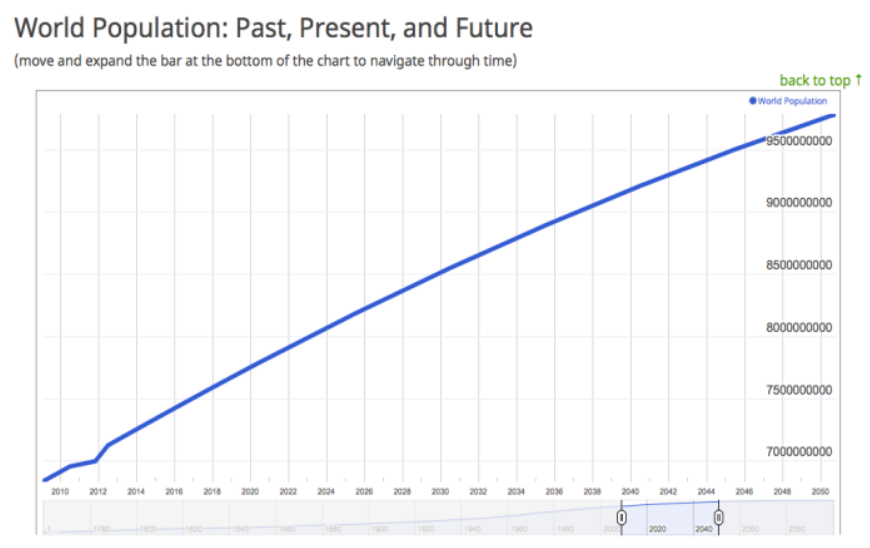

Fig. 1. The World Population taken from [1]. 


\section{MASLOW'S HIERARCHY OF NEEDS}

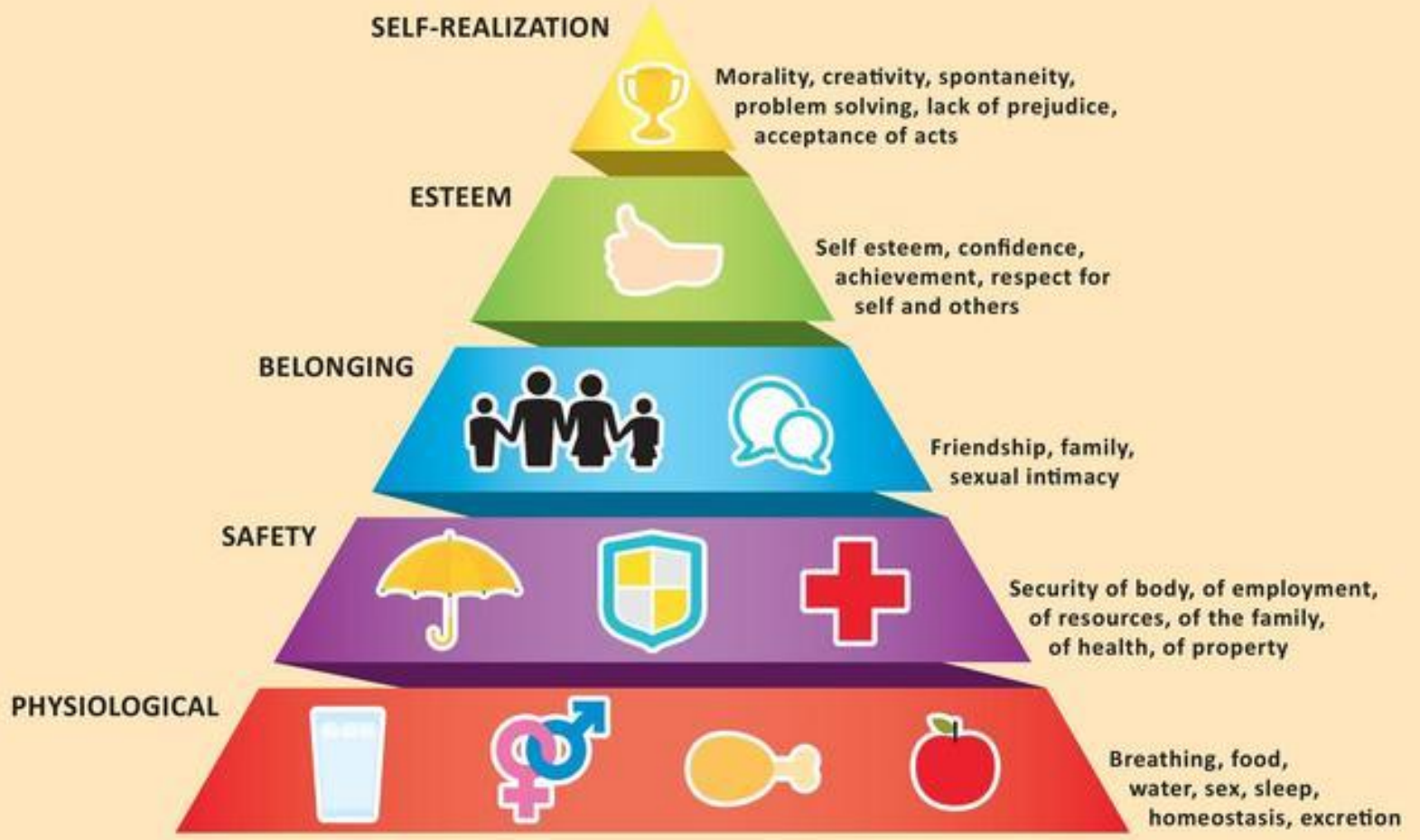

Fig. 2. The Maslow's Hierarchy of Needs taken from [6].

\section{DigITAL TECHNOLOGY: THE REVOLUTION}

It can be seen obviously that the world today is surrounded with digital technology massively. The digital technology is advancing continuously and being embraced by many industries. Figure 3 [7] illustrates the digital waves, the revolution of digital technology that we are experiencing today.

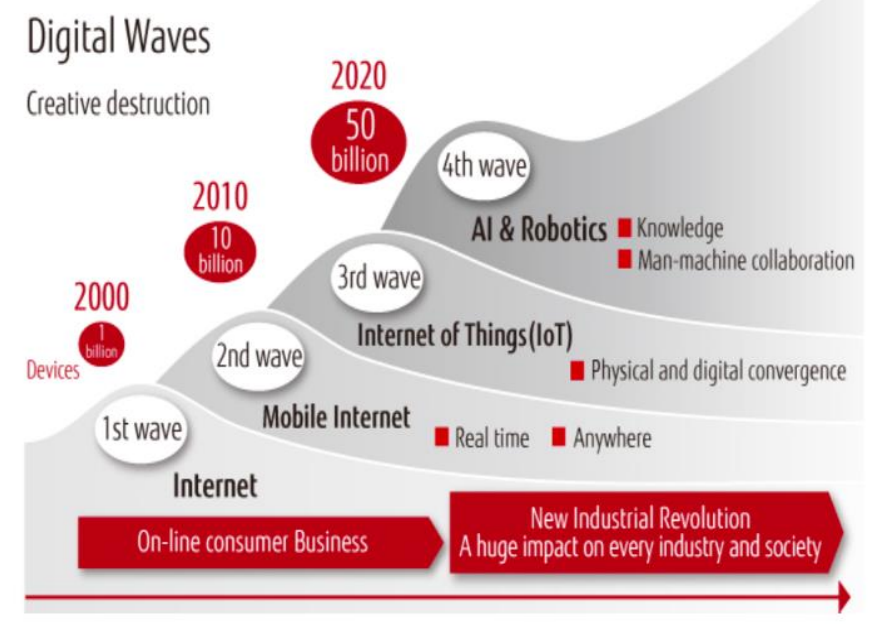

Fig. 3. The Digital Wave taken from [7].
The Internet was born during the first wave of digital technology, which give individual access and opportunity to wide variety of new and exciting borderless information and solution. Mobile Internet rose during the second wave of digital technology in which, there is an explosive growth of powerful smartphones and table devices. The world is now currently face the third wave of digital technology in which Internet of Things (IoT) emerged. It can be seen that the internet-enabled devices, expected to exceed 50 billion by 2020. IoT is about tiny embedded sensors and computers in equipment, machinery and devices, which can be link together and link to the cloud to generate new value for various industry and society. Robotics and Artificial Intelligence (AI) will be the main technology in the fourth wave of digital technology. It deals with the machine that is able to assist in knowledge creation process and decision making based on the combination of text and image data from the Internet as well as data from various sensors. The examples of modern digital technology are Cloud-based services, wearable sensors, biomarker detectors, mobile apps, the Internet of things, big data and AI [8].

As what can be seen now, IoT is one of the digital technology that surround today's daily live. IoT is an ecosystem of connected physical objects that are accessible through the Internet. These physical objects are connected using RFID, sensors/actuators and communication technologies[9]. 


\section{IOT FOR HEALTHCARE APPLICATION}

It is obvious that the initiative for embedding IoT in healthcare application is becoming a trend [10]. It is reported in [11], the IoT healthcare spending is expected to increase and total up to about $\$ 800$ billion in 2018. In general, the current application of IoT healthcare can be divided into two categories. The first one is for improvement of care while the second one is on tracking, monitoring and asset maintenance.

With regards to the first categories of application, which is improvement of care, there are two examples that will be shared in this paper. The first one is the IoT solution developed for Diabetic retinopathy. Diabetic retinopathy is a critical disease that is a leading cause for blindness in adult. It is quite a surprise to discover that the lifetime cost for the treatment is about USD1 million. However the cost will be way cheaper if it is treated early, which amount only USD50. The key to prevent this disease is to have an early detection in screening. Unfortunately, there is only $40 \%$ of patient that turn up for screening. In order to promote early detection, the Intelligent Retinal Imaging System (IRIS), LLC (limited liability company) of Pensacola has developed a testing solution screening which is accessible in rural and city area by using automated camera [12]. The solution works by sending the retina image captured by the automated camera to the clouds. The clouds have the IRIS developed algorithm for detecting the disease. The diagnosis report is then send back within 24 hours.

The second example of IoT healthcare application is continuous Glucose monitoring system [13]. The glucose monitoring system works by inserting the sensor to the body. The glucose data will be send wirelessly through transmitter to various display devices. With this solution, alert and alarm can be set in order to track the condition if the glucose level is heading high or low.

'AutoBed' is an example of the second categories of IoT healthcare application, which is tracking, monitoring and asset maintenance. 'AutoBed' is the prototype of 'smart bed' developed by the GE healthcare team collaborated with Mount Sinai Hospital, New York [14], [15]. Mount Sinai Hospital has more than 1000 beds and there are more than 59000 inpatients in a year. Hence, it can be concluded that there is a massive daily operation that take place in the hospital, specifically in assigning the patient to the available bed. 'AutoBed' is an IoT healthcare application prototype that can help in optimizing the process of assigning patient to available bed. Once the patient is admitted, the admitting nurse will enter all the required information of the patient into the electronic medical record form. The information includes the gender and 'triage' (the urgency of the case). Once the form is completed, then the bed request is triggered. The 'AutoBed' algorithm works by matching the information obtained from the electronic medical record form with the available bed in real-time using location awareness devices such as RFID, infrared and computer vision. With this innovation, it was found that the waiting time is decreasing by one-hour for more than $50 \%$ of incoming emergency room patient. In other words, it took about 7 calls to place a patient, but on the other hand, it is only 1 call with 'AutoBed'.

\section{ANALYTICS AND ITs Role In IoT APPLICATIONS}

Having IoT as a platform to generate the data alone is a waste. There is a major obstacle in making this an innovative solution to the healthcare services. It is true that with the IoT technology, fresh data from the patient is coming out and delivered easily to the healthcare provider. However, data without meaningful insight is meaningless. Many have reported that they face challenges when trying to interpret and transform these data into actionable oriented insights [16]. One of the challenges is the business owner or the technology provider need to apply some of the latest techniques of analytics to transform the data into actionable insight [16]. Therefore, the latest appropriate analytics are needed when developing the innovative products or services that will positively affect patient outcomes as well as deliver commercial growth.

It must be noted that it is hard for IoT solution to working efficiently without Analytics. Analytics is a scientific process of transforming data into insights for making better decisions. It is a multidisciplinary field that uses mathematics, statistics, predictive modeling \& machine learning techniques to discover meaningful pattern and knowledge. Analytics is a very significant field nowadays that surround today's daily life. Generally there are three types of analytics: Descriptive analytics, predictive analytics and prescriptive analytics [17].

Descriptive analytics is the most basic type. It serve to answer question like "what has happened?". Descriptive analytics analyze the real time incoming and historical data for having insight on how to approach the future. Basic statistics and mathematical techniques are among the most frequently used techniques for descriptive analytics. Figure 4 illustrates a screenshot of dashboard displaying heart rate and sleep data based on the resulted wearable sensors. It must be noted that the result displayed in the dashboard is obtained using descriptive analytics.

On the other hand, predictive analytics is a higher level of analytics that serve to answer question like "what could happen in the future based on previous trends and pattern?". It analyzes past data patterns and trends as well as tries to predict what could happen in the future. Among the frequent techniques that are used for predictive analytics are linear regression, multivariate regression, logistic regression, decision trees, random forest, naïve bayes and k-means. Figure 5 illustrates a screenshot of the Glucose monitoring system that used predictive analytics to predict the glucose level is heading low within 20 minutes.

Prescriptive analytics is an advanced analytics. It serves to answer question like "what should a business do?". Prescriptive analytics could advice on possible outcomes and results in action that is likely to maximize key business metrics. Optimization and simulation is among the famous techniques for prescriptive analytics.

IoT serves as a platform to obtain medical and health related data in healthcare application setting. Combining analytics with IoT will ensure all the relevant data is turned into actionable insight. As a matter of facts, IoT and analytics become a perfect combination for better healthcare solution. 


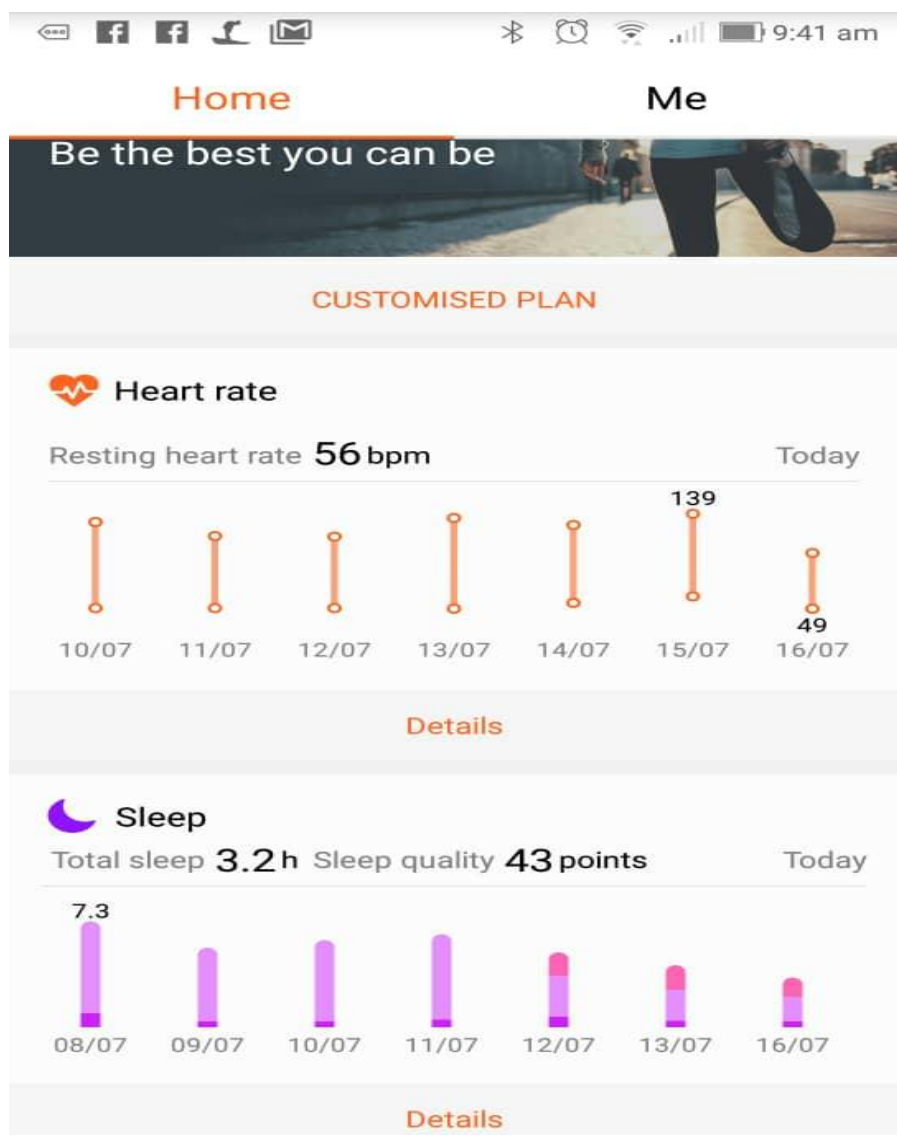

Fig. 4. An Example of Dashboard Displaying Result using Descriptive Analytics.

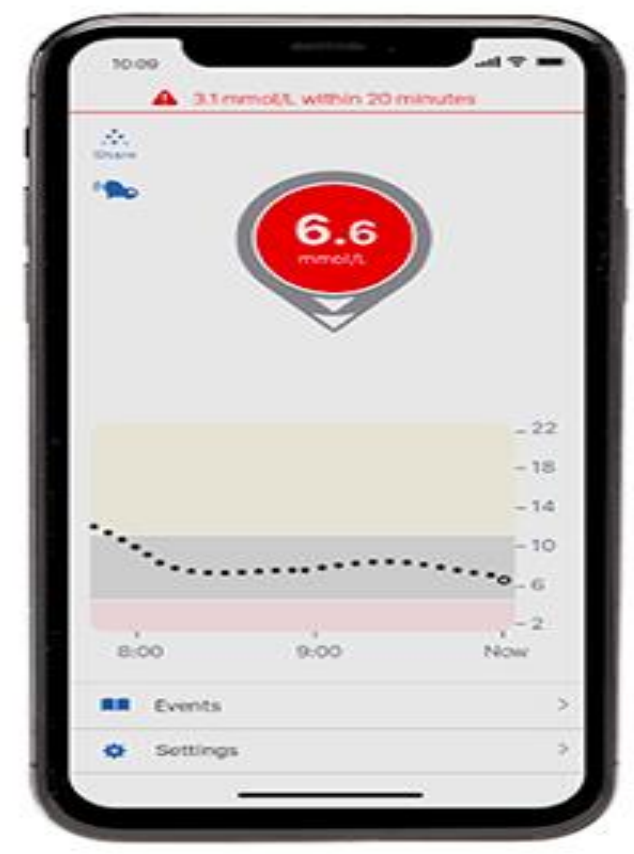

Fig. 5. An Example of Dashboard Displaying Result using Predictive, Analytics, taken from [13].

\section{DISCUSSION AND CONCLUSION}

Huge amount of medical data are available through this third digital wave of technology, which is IoT. It should also be noted that the amount of data from the utilization of this technology is increasing exponentially. As a matter of facts, analytics offers better way to quickly provides relevant insights using this data.

From the healthcare perspective, the wide use of IoT devices such as wearable sensors as well as the mobile data analysis application will transform the patient from 'passive patients' to 'active patients'. Passive patients refer to patient that relies heavily on the doctors or the healthcare providers when it comes to addressing the health issues. On the other hand, active or activated patient refer to patient that take matters into their own hands. Take for example, a person or patient with wearable device such as fitbits, or those with at home monitor for glucose or blood pressure level and also those with mobile applications that transmit health related data. This example of patients will monitor and control their own health instead of just waiting for the regular check-up. Therefore, there is an active participation in the personalized healthcare, which leads to increase engagement in preventive care and also increase effectiveness of health practices.

One of the challenges that might be faced when adopting this technology is to ensure that all patients have equal opportunity to access this technology and get benefit from it. Therefore, it is important for all parties like government, health human resource and technology provider companies to work together for making this technology accessible to all. A solid policy in adopting this technology should be carefully formulated in order to ensure it is beneficial to all.

Although it is projected that the IoT healthcare analytics will soon be the technology that is widely adopted, it should be highlighted here that IoT healthcare analytics would never replace the health human resource. The IoT healthcare analytics technology could never be equal to human. Rather, it only complements the health human resource by providing data from sensors for better diagnoses and treatment plan. Furthermore, it is able to reduce inefficiencies and waste in healthcare system. In some cases, health human resource could focus in more significant responsibilities. Therefore, health human resource should never feel threaten by adopting the technology (like IoT, artificial intelligence, robots etc) as 'human touch' is still a top priority in delivering healthcare services.

\section{ACKNOWLEDGMENT}

Special thanks to Ministry of Education Malaysia for granting a grant TRGS/1/2016/FKP-AMC/01/D00005 and Universiti Teknikal Malaysia Melaka.

[1] "Current world population." [Online]. Available: http://www.worldometers.info/world-population/. [Accessed: 27-Jul2018].

[2] "Impact of migration on population change," European Union, 2013. [Online]. Available: https://www.espon.eu/sites/default/files/attachments/demifer_PB_migr_i mpact.pdf.

[3] S. L. Colby and J. M. Ortman, "Projections of the size and composition 
of the U.S. population: 2014 to 2060 Population estimates and projections," 2015.

[4] "World population projected to reach 9.8 billion in 2050, and 11.2 billion in 2100," United Nations. [Online]. Available: https://www.un.org/development/desa/en/news/population/worldpopulation-prospects-2017.html. [Accessed: 27-Jul-2018].

[5] D. Ramanayake, V. Giovanni, B. Morgagni, and F. Fi, "Critical review of growing population and climate challenges: How to effects the future social structural changes and world conflict in 2100," Int. J. Dev. Res., vol. 08, pp. 20133-20139, 2018.

[6] "Maslow's Pyramid." https://www.vecteezy.com/vector-art/91591-maslow-s-pyramid.

[7] "How digital technology will transform the world: The digital transformation has arrived," Fujitsu Journal, 2016. .

[8] L. Li, "China' s manufacturing locus in 2025 : With a comparison of “ Made-in-China," Technol. Forecast. Soc. Chang., no. May, pp. 0-1, 2017.

[9] S. Madakam, R. Ramaswamy, and S. Tripathi, "Internet of Things ( IoT ): A Literature Review," J. Comput. Commun., vol. 3, no. May, pp. 164$173,2015$.

[10] H. H. Nguyen, F. Mirza, M. A. Naeem, and M. Nguyen, "A review on IoT healthcare monitoring applications and a vision for transforming sensor data into real-time clinical feedback," in 2017 IEEE 21st International Conference on Computer Supported Cooperative Work in Design (CSCWD), 2017, pp. 257-262.

[11] V. Velamoor et al., "Unlocking digital health: Opportunities for the mobile value chain," 2015.

[12] "From the person, to the cloud, and back: How remote patient monitoring and intelligent feedback loops are driving the personalization of care," 2017.

[13] "Dexcom Continuous glucose monitoring." [Online]. Available: https://www.dexcom.com/continuous-glucose-monitoring.

[14] "How bed-tracking technology allowed Mt. Sinai Medical Center to reduce admission wait times," 2013. [Online]. Available: https://www.beckershospitalreview.com/healthcare-informationtechnology/how-bed-tracking-technology-allowed-mt-sinai-medicalcenter-to-reduce-admission-wait-times.html.

[15] B. G. Thomas et al., "Automated bed assignments in a complex and dynamic hospital environment," Interfaces (Providence)., vol. 43, 2013.

[16] I. Lee and K. Lee, "The Internet of Things ( IoT ): Applications , investments , and challenges for enterprises," Bus. Horiz., vol. 58, no. 4, pp. 431-440, 2015.

[17] S. Ransbotham, D. Kiron, and P. K. Prentice, "Minding the analytics gap,” MIT Sloan Manag. Rev., 2015. 